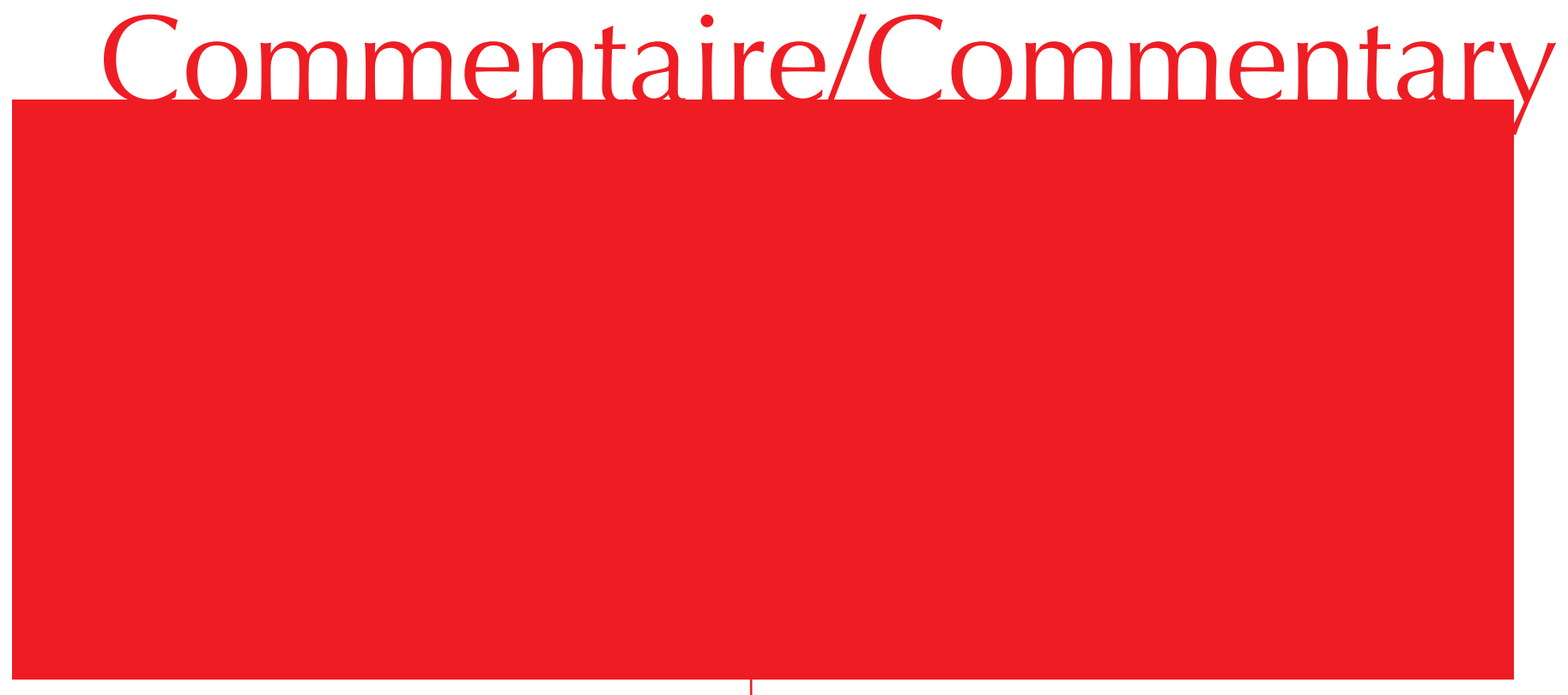

\section{Mobilisation d'infirmières et d'infirmiers dans la foulée du printemps érable : genèse, réflexion et perspectives futures}

\section{KARINE PHILIBERT, KAROLINE DEMERS, SOPHIE VALLÉE-DESBIENS, \& DOMINIQUE BILODEAU}

\section{Introduction}

Le 17 février 2012, Sophie Desbiens, étudiante finissante à I'École des sciences infirmières de I'Université de Sherbrooke, crée sur le réseau social Facebook un groupe qu'elle intitule Infirmières et infirmiers contre la hausse et qu'elle décrit comme suit :

Ce groupe représente un lieu de rassemblement virtuel pour tous les infirmières et infirmiers qui s'opposent à la hausse des droits de scolarité de $1625 \$$ que le gouvernement Charest veut imposer à la communauté étudiante universitaire. Il est aussi ouvert aux étudiantes et étudiants en soins et sciences infirmières.

Le niveau d'instruction des populations étant reconnu par l'OMS comme un déterminant significatif de la santé, nous pensons qu'il est du devoir des infirmières et des infirmiers de s'opposer à toutes mesures pouvant limiter l'accès à l'éducation. Les infirmières et les infirmiers ont un rôle fondamental à jouer dans la défense des valeurs de justice et d'équité sociales. Tous les jours, nous travaillons auprès de personnes vulnérables et marginalisées et nous sommes aux premières loges pour constater les ravages des politiques gouvernementales ayant pour effet d'accroître les inégalités sociales : maladies chroniques, manque d'accessibilité aux soins, problèmes de santé mentale, effritement du tissu social, etc. Soigner ne suffit plus : nous devons prendre position sur la place publique afin de dénoncer le contexte sociopolitique qui contribue à ces problématiques.

Par ailleurs, étant donné que le baccalauréat en sciences infirmières devrait, au cours des prochaines années, devenir 
obligatoire à l'exercice de la profession infirmière au Québec, nous craignons que la hausse des frais de scolarité ne limite I'accessibilité à ces programmes de formation universitaires. Nous sommes déjà en contexte de pénurie. Faut-il ajouter un obstacle supplémentaire à ceux et celles qui souhaitent joindre les rangs de la profession ? Parce que la hausse des frais de scolarité relève d'une logique économique où l'éducation est reléguée au rang de marchandise et où l'être humain n'a de valeur que dans la mesure où il est « productif », nous devons nous unir pour défendre les valeurs de dignité, d'équité, de justice sociale et de solidarité si chères à notre profession. »

\section{Hausse des frais de scolarité : des conséquences sociales graves}

Plus ou moins à l'aise avec les règles du réseau social, Sophie Desbiens y inscrit tous ses " amis » infirmières et infirmiers. Plusieurs se désabonnent aussitôt, créant l'impression que le groupe ne survivra pas longtemps. Toutefois, ceux qui demeurent abonnés se mettent à discuter et à inviter d'autres amis et collègues à se joindre à eux. Il y a des infirmières et infirmiers, mais aussi des ambulanciers, quelques médecins et plusieurs étudiants du milieu de la santé. On y discute de la crise actuelle, de l'éducation et de l'impact des politiques néolibérales sur la santé. À mesure que le conflit se prolonge et se complique, l'urgence de s'impliquer concrètement se fait sentir. Le groupe réalise que plusieurs soignants apportent déjà dans les manifestations des trousses de premiers soins, au cas où certains manifestants ou passants seraient blessés.

Le groupe contacte la CLASSE (Coalition Large de I'Association pour une Solidarité Syndicale Étudiante) et la FIQ (Fédération Interprofessionnelle du Québec) afin $d^{\prime}$ offrir ses services en tant que médi-militant, un terme que la majorité des infirmières et infirmiers contre la hausse entend pour la première fois. Agréablement surpris par notre initiative, les deux syndicats nous fournissent trousses de premiers soins, radios $\mathrm{CB}$ et dossards. La première action médi-militante concertée a lieu à Montréal le 22 mars 2012; nous serons une vingtaine d'infirmières et infirmiers à $y$ participer. La manifestation est pacifique, familiale, et les rares interventions visent des personnes légèrement déshydratées. Parce que tout se déroule bien, d'autres évènements sont envisagés avec enthousiasme. Le 14 avril 2012, le groupe participe donc à une deuxième manifestation à Montréal. Par la suite, les membres du groupe décideront de se rendre à Victoriaville pour un rassemblement majeur en marge du Congrès du Parti libéral du Québec (PLQ).

Nous sommes six de trois villes différentes à nous rejoindre à Victoriaville. En chemin vers I'hôtel le Victorin où se déroule le Congrès du PLQ, en compagnie des 3000 autres manifestants de tous âges, nous blaguons sur l'utilité de nos trousses de premiers soins qui n'ont pas encore vraiment servi. Mais nous prenons soin de nous rappeler qu'il nous faut demeurer vigilants car les manifestations ont toujours le potentiel de « mal tourner».

Faut-il revenir sur les évènements de Victoriaville ? Pendant que le congrès du PLQ bat son plein malgré les odeurs de gaz lacrymogène qui s'infiltrent par le système de ventilation de I'hôtel, et qu'à Québec les syndicats et la Ministre de l'éducation tentent de trouver une issue à la crise, c'est le chaos dans les rues de Victoriaville. Qui a jeté la première pierre, qui a tiré le premier nuage de gaz ? Comment se fait-il que personne n'ait entendu l'annonce selon laquelle la manifestation était illégale ? Si cet appel avait été entendu, est-ce que des familles entières seraient demeurées immobiles face aux policiers de la Sûreté du Québec alors que ceux-ci se préparaient à déployer leur arsenal de gaz et de balles de plastique? À peine quelques minutes après le début de la démonstration, de nombreux effluves de gaz lacrymogènes jaillissent sur les manifestants, incommodant au passage plusieurs enfants et personnes âgées. Cela constituera un prélude à un des plus violents face-à-face entre civils et forces de l'ordre dans I'histoire du Québec moderne. Un membre de notre groupe décrit son expérience en ces termes :

" Je suis à genoux dans le gazon. Je n'ai pas le temps de mettre mes gants, j'ai du sang sur les doigts. L'eau ruisselle partout, du gaz coule sur mon visage, ça brûle. Des voix, de la fumée, des gens qui courent. Une clameur résonne sans cesse: « Il y a un blessé, il y a un blessé! ». Un mouvement de panique naît et la foule se presse autour de la victime. J'évalue son état : saignement, traumatisme crânien, état de conscience altéré, condition précaire. Je donne les premiers soins. Ma radio $C B$ ne fonctionne plus, je n'ai pas de contact avec mes collègues. Vont-ils me trouver, ici, au milieu de la foule, dans un nuage de gaz et de cris? Je me sens tout à coup vulnérable. Un sentiment de panique momentané, vite évacué : j'en ai vu d'autres, je reste en contrôle. L'adrénaline rougit mes joues et canalise mon énergie. On attend l'ambulance mais c'est la confusion : elle refuse de venir ou on lui bloque l'accès, personne ne comprend ce qui se passe. On avance sur nous : la foule, mais aussi les forces de l'ordre. Des gaz lacrymogènes sont lancés en notre direction, on étouffe. Je croise du regard les autres infirmières : il faut déplacer le blessé immédiatement. Je dirige la manœuvre. On transporte péniblement le jeune 
homme à bout de bras mais de multiples facteurs nuisent à I'opération : des souches, un fossé, le sol mouillé et glissant. Des policiers nous dévisagent, le regard vide et les bras pendants, visiblement dépassés par les événements. Aucune aide n'est offerte. Et l'ambulance qui n'arrive pas! Je ressens une profonde colère, une impuissance insoutenable. Lorsque le jeune blessé quitte finalement les lieux, je me sens seule, terriblement seule et le cœur vide. Je suis en état de choc, j'ai besoin d'air. Je veux agir, crier que notre jeunesse est belle et qu'il faut la protéger et l'aimer, non pas lui tirer dessus pour la faire taire. Je marche, la mine défaite et l'esprit centré sur la tâche à venir. On me dévisage, je me dis que je dois avoir I'air d'une morte. Je jette un coup d'œil à mon uniforme : il est maculé de sang. »

Cette soirée-là, nos trousses de premiers soins auront bel et bien servi... jusqu'à ce que le matériel nous manque pour soigner les blessés. Nous avons soigné encore une lacération de I'œil (organe qui sera finalement " perdu » après des heures de chirurgie d'urgence à l'hôpital); une hémorragie fémorale et des dizaines d'autres blessures. Nous vivrons, durant ces longues heures, des émotions fortes oscillant entre abjection et impuissance. Ces émotions nous habiterons pendant plusieurs jours.

Afin de canaliser celles-ci de manière constructive, notre collectif a accepté I'invitation d'un député afin de participer à une conférence de presse exigeant une enquête indépendante sur les évènements de Victoriaville. Il s'agit de la première sortie publique $d^{\prime}$ Infirmières et d'infirmiers contre la hausse. Les évènements que nous venons de vivre nous ont rendus plus solidaires et déterminés. Deux infirmières parlent au nom du groupe, mais les autres sont présents dans la salle pour les soutenir. Nos réponses aux questions des journalistes demeurent très prudentes : nous ne voulons pas être accusés d'avoir participé à une manifestation illégale. En accord avec notre code de déontologie professionnelle, nous refusons de dévoiler l'identité des blessés et d'émettre notre avis sur l'origine des blessures, dans l'espoir qu'une enquête indépendante permette à des experts de se prononcer sur les projectiles utilisés.

Or, I'enquête n'aura pas lieu. Et pourtant, pour celles et ceux qui se trouvaient sur le terrain, les balles de plastiques recueillies auprès des blessés et les ecchymoses observées - à la fois profondes et à la circonférence bien délimitée ne laissent aucun doute sur la nature des projectiles ayant provoqué certaines des blessures les plus graves. Il ne s'agit pas de roches ou de balles de billards. Sans nul doute, Victoriaville nous aura catapultés aux premières loges du conflit qui secoue tout le Québec depuis plusieurs mois. La conférence de presse, quant à elle, nous aura convaincus de la pertinence de faire entendre nos voix d'infirmières et $d^{\prime}$ infirmiers et ce, sur la place publique, afin de témoigner de ce que nous avons observé à titre d'experts de la santé. Nous estimons qu'une nouvelle génération d'infirmières et d'infirmiers se doit de refuser de se contenter du rôle d'exécutant qui lui est assigné et qu'elle doit oser exposer les nombreux problèmes qui affectent notre système de santé et la santé de tous.

\section{Conclusion}

Suite aux évènements, de Victoriaville et aussi en prévision de ce que l'automne 2012 nous réserve lors de la rentrée étudiante, nous nous sommes réunis afin de discuter de l'avenir du groupe. Nous avons décidé de poursuivre notre médi-militantisme et avons accepté l'invitation de la CLASSE $d^{\prime}$ offrir un atelier de premiers soins lors $d^{\prime}$ une fin de semaine de formation. Nous projetons par ailleurs de presser la FIQ $d^{\prime}$ envisager une grève sociale générale $d^{\prime}$ une journée comme moyen de pression contre le gouvernement libéral de Jean Charest. Nous pensons éventuellement rebaptiser le groupe sous le vocable: "Collectif des infirmières et infirmiers pour la justice sociale », question d'assurer sa pérennité au-delà du conflit étudiant secouant le Québec. D'autres causes nous interpellent : la privatisation du système de santé, les attaques de membres du gouvernement Harper au droit à l'avortement... la liste est longue. Pour nous, ce n'est qu'un début, un apprentissage difficile sur le terrain de la démocratie et de ses liens étroits, voire vitaux, avec la santé des citoyennes et des citoyens du Québec.

Karine Philibert, inf., B.Sc.Inf.

Karoline Demers, inf., B.Sc.Inf.

Sophie Vallée-Desbiens, inf., B.Sc.Inf.

Dominique Bilodeau, inf.

Infirmières contre la hausse des frais de scolarité http://www.facebook.com/groups/316905925030141/ 